\title{
The Characteristics of Complex Chen CHAOTIC Systems with Time-Delay and Its Self-Delay Synchronization Control
}

\author{
Sen Leng ${ }^{1}$, Jiaxun Liu ${ }^{1}$, Fangfang Zhang ${ }^{1,2^{*}}$ and Zhengfeng $\mathrm{Li}^{1}$ \\ ${ }^{1}$ College of Electrical Engineering and Automation, Qilu University of Technology (Shandong Academy of Sciences), Jinan \\ 250353, China \\ ${ }^{2}$ Qilu University of Technology (Shandong Academy of Sciences), Shandong Computer Science Center \\ (National Supercomputer Center in Jinan), Shandong Artificial Intelligence Institute, Jinan 250101, China \\ *Corresponding author
}

\begin{abstract}
As for chaotic systems with time-delay, different studies have been done on chaotic communication. However, complex Chen system with time-delay have not been investigated so far, although it has potential applications in many important fields. Therefore, we first explore the natures of complex Chen chaotic system with time-delay, and study its self-delay synchronization. Firstly, a complex Chen system with time delay is introduced, and its chaotic characteristics are analyzed. Secondly, according to the error feedback principle, the self-delay synchronization controller is designed. Finally, the validity of the controller is verified by mathematical proof and computer simulation. Simulation results show that the controller is simple and easy to be realized in practice.
\end{abstract}

Keywords-complex chaotic system; time-delay; self-delay synchronization controller; lyapunov index

\section{INTRODUCTION}

Due to pseudorandom characteristics of chaos, chaotic synchronization is especially suitable for secure communication [1]. Therefore, chaotic systems with time-delay are better than systems without time-delay in description of real physical processes. Moreover, a time-delay chaotic system with multiple positive Lyapunov exponents is an infinite dimensional system. It has more complicated dynamic characteristics than ordinary chaotic systems. Therefore, the cryptogram formed by adding time-delay chaotic signal has better security. Time-delay chaotic system becomes a hot issue in current chaotic secure communication researchs [2-5].

In 1977, Mackey and Glass[6] first found that chaos exists in the delay system, when studied the first-order nonlinear delay differential equation describing the physical control system. And then people begin to pay close attention to time delay chaotic systems. Pyragas [7] used the delay differential equations to prove that chaotic system with time-delay can transmit signals. J. Sun [8] studied chaotic synchronization of systems with time delays by means of unidirectional linear error feedback coupling and established the chaotic synchronization of time delays in the transmission channel of common conditions. J. Tang et al. [9] applied time varying delay to Chen chaotic system. The delay synchronization

problem is solved successfully. Researchers have not studied the complex Chen system with time-delay. This paper first applied time-delay to complex Chen system.

In actual engineering, due to machinery friction, noise, vibration, etc., the changes of the object are often affected by the past state and the current state. The actual system and the expected system are not synchronized. We keep the system structure and parameters unchanged, and make the time-delay system synchronize with the original system. We call this synchronization self-delay synchronization [10]. The self-delay synchronization makes the practical system maintain the performance as well as the original. Therefore, it is of great significance in theory and in reality.

The complex Chen system is widely used in various fields. Therefore, the characteristics of time-delay complex Chen system and its self-delay synchronization control are studied. The rest of this paper is as follows: Section II studies the characteristics of complex Chen system with one time-delay. We design self-delay synchronization controller and give a rigorous mathematical proof in Section III. Section IV makes simulation with MATLAB. Finally, we summarize the full text in Section V.

\section{TIME DELAy COMPLEX CHEN SYSTEM}

\section{A. Chaotic Characteristics}

The complex Chen system is:

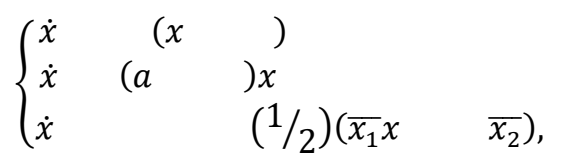

where $x$ variables, and real and virtual parts of equations (1): are the complex is the real state variable. Separate the 


$$
\left\{\begin{array}{l}
\dot{u}_{1}=a_{1}\left(u_{3}-u_{1}\right) \\
\dot{u}_{2}=a_{1}\left(u_{4}-u_{2}\right) \\
\dot{u}_{3}=\left(a_{2}-a_{1}\right) u_{1}-u_{1} u_{5}+a_{2} u_{3} \\
\dot{u}_{4}=\left(a_{2}-a_{1}\right) u_{2}-u_{2} u_{5}+a_{2} u_{4} \\
\dot{u}_{5}=-a_{3} u_{5}+\left(u_{1} u_{3}+u_{2} u_{4}\right),
\end{array}\right.
$$
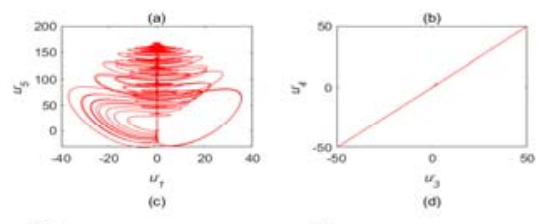

it is a $5 \mathrm{D}$ chaotic autonomous system.

Consider the following delayed complex Chen systems:

$$
\left\{\begin{array}{l}
\dot{y}_{1}=a_{1}\left(y_{2}-y_{1}\right) \\
\dot{y}_{2}=\left(a_{2}-a_{1}\right) y_{1}+a_{2} y_{2}-y_{1} y_{3}(t-\tau) \\
\dot{y}_{3}=-a_{3} y_{3}(t-\tau)+(1 / 2)\left(\overline{y_{1}} y_{2}+y_{1} \overline{y_{2}}\right),
\end{array}\right.
$$

where $0 \leq \tau \leq \tau_{m}$ is the delay factor, $y_{1}=u_{1}{ }^{\prime}+j u_{2}{ }^{\prime}, y_{2}=$ $u_{3}{ }^{\prime}+j u_{4}{ }^{\prime}$ are the complex state variables of the system, and $y_{3}=u_{5}{ }^{\prime}$ is the real state variable. The system (1) is a singledelay complex Chen system. The real and virtual parts of the separation equations (3) are:

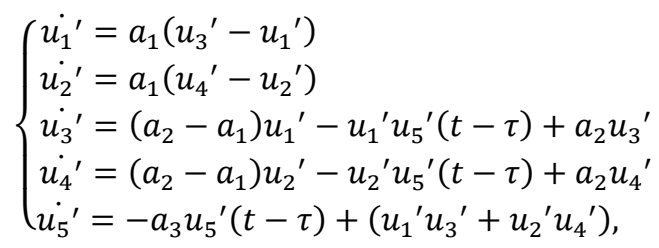

Therefore, the dissipation and symmetry of the original system (2) are still valid for the system (4), but the chaotic characteristics of the time-delay system are quite different from those of the original system. The system (4) produces highly stochastic and unpredictable time series with chaotic projection planes and projection spaces with different time-delay factors. Attractors are shown in Figure 1 and Figure 2.

\section{B. Initial Value Sensitivity}

Let $\tau=1 \mathrm{~s}$, select two very similar initial values, such as $(2,1,3,4,2)^{T}$ and $(2.001,1.001,3.001,4.001,2.001)^{T}$. The state evolution of the system is shown in Figure 3. We know that the time-delay system is sensitive to the initial state value from the graph.

\section{Lyapunov Index Calculation}

According to the calculation method [11] of Lyapunov index we get the Lypaunov exponent schematic diagram of the single delay complex Chen system with the different time delay factor $\tau$, as shown in Figure 4. Table 1 shows some values of Lyapunov exponent. Obviously, the characteristics of the single-delay complex Chen system and the positive Lyapunov index are related to the $\tau$. It also shows the randomness and unpredictability of the time-delay system due to the $\tau$.

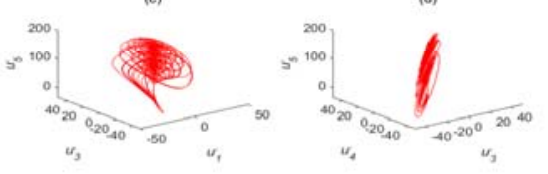

FIGURE I. ATTRACTOR OF SYSTEM (2)( T = 1S).
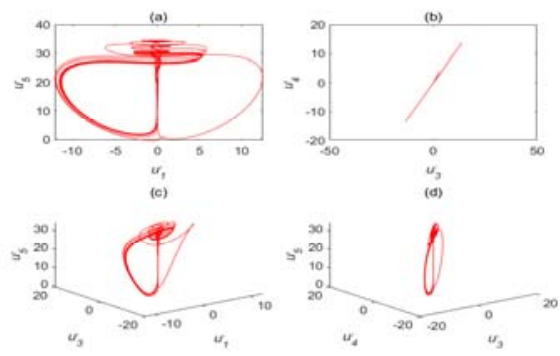

FIGURE II. ATTRACTOR OF SYSTEM $(2)(\tau=2(\cos t+\operatorname{rand}(\mathrm{t})) \mathrm{s})$.



FIGURE III. INITIAL SENSITIVITY OF COMPLEX CHEN SYSTEMS WITH TIME DELAY.

\section{SELF-DELAY SYNCHRONOUS CONTROLLER}

Firstly, the definition and related lemma of self-delay synchronization for complex chaotic systems are given. And then the self-delay synchronization controller is designed for single-delay complex Chen chaotic systems.

\section{A. The Related Theorem}

Definition 1 [10] First give the following complex chaotic system:

$$
\left\{\begin{array}{l}
\dot{X}(t)=f(X(t)) \\
\dot{Y}(t)=f(Y(t-\tau))+V(Y(t-\tau), Y(t)),
\end{array}\right.
$$

where $\quad X(t)=\left\{x_{1}(t), x_{2}(t), \cdots, x_{n}(t)\right\}^{T} \quad$ and $\quad Y(t)=$ $\left\{y_{1}(t), y_{2}(t), \cdots, y_{n}(t)\right\}^{T}$ are complex state vector, $\tau=$ $\left\{\tau_{1}, \tau_{2}, \cdots, \tau_{n}\right\}^{T}\left(\tau_{i} \geq 0, i=1,2, \cdots, \mathrm{n}\right)$ is the delay factor vector. Here $\dot{X}(t)=f(X(t))$ is the original system and $\dot{Y}(t)=$ $f(Y(t-\tau))+V(Y(t-\tau), Y(t))$ is the time-delay system. 
If there is a controller v satisfying:

$$
\begin{aligned}
& \lim _{t \rightarrow \infty}\|Y(t)-X(t)\|^{2}=\lim _{t \rightarrow \infty}\left(\left\|Y(t)^{r}-X(t)^{r}\right\|^{2}+\| Y(t)^{i}-\right. \\
& \left.X(t)^{i} \|^{2}\right)=0,
\end{aligned}
$$

then we call it as self-delay synchronization between $X(t)$ and $Y(t)$.

Lemma 1 [12]: For linear continuous time-delay systems:

$$
\dot{Z}(t)=A Z(t)+B Z(t-\tau)(\tau \geq 0),
$$

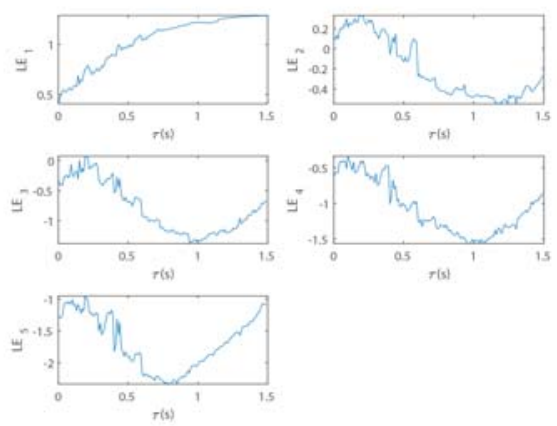

FIGURE IV. LYPAUNOV INDEX SCHEMATIC DIAGRAM OF TIMEDELAY COMPLEX CHEN SYSTEM.

where $\mathrm{Z}(\mathrm{t}) \in \mathrm{R}^{n}, \mathrm{~A}, \mathrm{~B}$ are $\mathrm{n} \times \mathrm{n}$ constant matrices. If there is a positive definite matrix $P, M$ make the following matrix negative definite:

$$
\left(\begin{array}{cc}
A^{T} P+P A+M & \frac{P B+B^{T} P}{2} \\
\frac{P B+B^{T} P}{2} & -M
\end{array}\right) .
$$

Then $\mathrm{Z}(t)=0$ is the global exponentially asymptotically stable equilibrium point of the system (5)

\section{B. Design Controller}

Considering the original system (1) and the single-delay complex Chen system (3), the following controller is applied to the system (3):

$$
\left\{\begin{array}{l}
v_{1}=k_{1} e_{1} \\
v_{2}=k_{2} e_{2} \\
v_{3}=u_{1}^{\prime} u_{5}^{\prime}(t-\tau)-u_{1} u_{5}+k_{3} e_{3} \\
v_{4}=u_{2}^{\prime} u_{5}^{\prime}(t-\tau)-u_{2} u_{5}+k_{4} e_{4} \\
v_{5}=-\left(u_{1}^{\prime} u_{3}^{\prime}+u_{2}^{\prime} u_{4}^{\prime}-u_{1} u_{3}-u_{2} u_{4}\right)+k_{5} e_{5}
\end{array}\right.
$$

where $k_{i} \in R^{n}, i=1,2, \cdots, 5$, and if $k_{1}=k_{2}=0$, then there are $k_{3}, k_{4}$ and $k_{5}$ such that

$$
\lim _{t \rightarrow \infty} \sum_{i=1}^{5}\left(u_{i}{ }^{\prime}-u_{i}\right)^{2}=0
$$

It is said that the complex Chen system (1) and the single-
delay complex Chen system (3) realize self-delay synchronization.

\section{Prove}

Let $e_{i}(t)=u_{i}{ }^{\prime}(t)-u_{i}(t), e_{i}(t-\tau)=u_{i}{ }^{\prime}(t-\tau)-u_{i}(t)$, $i=1,2, \cdots, 5$, delay error systems for obtainable systems (2) and systems (4):

$$
\begin{gathered}
\left\{\begin{array}{l}
\dot{e}_{1}=a_{1}\left(e_{3}-e_{1}\right) \\
\dot{e}_{2}=a_{1}\left(e_{4}-e_{2}\right) \\
\dot{e}_{3}=\left(a_{2}-a_{1}\right) e_{1}+\left(k_{3}+a_{2}\right) e_{3} \\
\dot{e}_{4}=\left(a_{2}-a_{1}\right) e_{2}+\left(k_{4}+a_{2}\right) e_{4},
\end{array}\right. \\
\dot{e}_{5}=-a_{3} e_{5}(t-\tau)+k_{5} e_{5} .
\end{gathered}
$$

Error system (11) is a linear time-invariant system. If appropriate $k_{3}$ and $k_{4}$ are selected to make all eigenvalues have negative real parts, the error system (11) is exponentially asymptotically stable. Error system (12) is a special case of linear continuous time-delay system (8) where $\mathrm{A}=k_{5}, B=$ $-a_{3}$.

According to Lemma 1 , if there are positive number $p, m$ which make

$$
\left(\begin{array}{cc}
2 k_{5} p+m & -p a_{3} \\
-p a_{3} & -m
\end{array}\right)
$$

negative definite ,then $e_{5}=0$ is the global exponentially asymptotically stable equilibrium point of the system (7).

In summary, the error system (11)-(12) are globally exponentially asymptotically stable at zero, and the controller is established.

\section{SIMULATION}

The original Chen system (2) is used as the driving system and the system (4) is used as the response system. The initial values of the system are $\mathrm{u}(0)=(1,2,3,4,1)$ and $\mathrm{u}^{\prime}(0)=$ $(-1,-2,-3,-4,1)^{T}$ and $\tau=1$ s, respectively. Let $k_{3}=k_{4}=$ $k_{5}$, by using the controller shown in (10), we obtain the eigenvalues of the error system (11) respectively. For the error system (12), there obviously exist positive numbers $p, m$ (such as $p=1, m=201$ ) that makes the following equations true.

$$
\left\{\begin{array}{l}
-200 p+m<0 \\
200 p m-m^{2}-64 /{ }_{9} p^{2}>0 .
\end{array}\right.
$$

The error diagram of the self-delay synchronization is shown in Figure 6, and $t$ represents the time. The simulation results are consistent with the theoretical analysis. The validity of the controller is verified. The controller has fast convergence with suitable $k_{3}=k_{4}=k_{5}$. Compared with other synchronization controllers, we only employ part of the state variables and realize the synchronization of all variables. 
[3] F. Zhang, "Complete Synchronization of Coupled Multiple-time-delay Complex Chaotic System with Applications to Secure Communication,” Acta Physica Polonica B, vol. 46, no. 8, p. 1473, 2015.

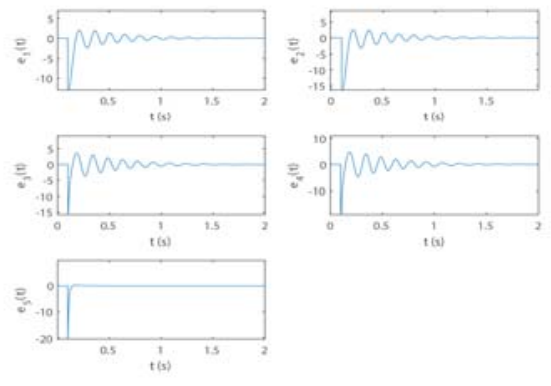

FIGURE V. STATE ERROR DIAGRAM OF SELF-DELAY SYNCHRONOUS SYSTEM.

TABLE I. PARTIAL VALUE OF LYPAUNOV EXPONENT

\begin{tabular}{|c|c|c|c|c|c|}
\hline$\tau / \mathrm{s}$ & $\mathrm{LE}_{1}$ & $\mathrm{LE}_{2}$ & $\mathrm{LE}_{3}$ & $\mathrm{LE}_{4}$ & $\mathrm{LE}_{5}$ \\
\hline 0.01 & 0.4048 & 0.1111 & -0.3290 & -0.6287 & -1.2673 \\
\hline 0.10 & 0.5442 & 0.2977 & -0.0610 & -0.5120 & -1.0797 \\
\hline 0.50 & 0.9820 & -0.1092 & -0.6741 & -0.9983 & -1.8741 \\
\hline 1.00 & 1.2215 & -0.4859 & -1.3462 & -1.5042 & -2.0572 \\
\hline 1.50 & 1.2881 & -0.2633 & -0.6733 & -1.8557 & -1.0835 \\
\hline
\end{tabular}

\section{CONCLUSION}

In this paper, we study one time-delay complex Chen chaotic system, and analyze its chaotic characteristics including dissipation, symmetry, initial value sensitivity, Lyapunov exponent and so on. Then we design the controller to realize the self-delay synchronization of one time-delay complex Chen system and give a rigorous mathematical proof. Finally, the simulation experiments are made, and all error functions converge to zero quickly. The results are consistent with the theoretical analysis. The principle of the controller is simple and and clear to implement.

Besides, system of time-delay complex chaotic can produce various pseudorandom series. These unpredictable and reproducible time series can be used in chaotic secure communication to improve the security performance.

\section{ACKNOWLEDGMENT}

The article is supported by Young Doctorate Cooperation Fund Project of Qilu University of Technology (Shandong Academy of Sciences) (No. 2018BSHZ001), International Collaborative Research Project of Qilu University of Technology (No. QLUTGJHZ2018020), National Nature Science Foundation of China (No. 61603203 and 61773010), and Nature Science Foundation of Shandong Province (No. ZR2017MF064).

\section{REFERENCES}

[1] J. Liu, Z. Wang, M. Shu, F. Zhang, S. Leng, and X. Sun, "Secure communication of fractional complex chaotic systems based on fractional difference function synchronization," Complexity, vol. 2019, 2019.

[2] C. Chen, "Advances in the application of modern information security and chaotic secure communication,” Information Recording Materials, vol. 19, no. 9, pp. 8-10, 2018.
[4] B. Sun, M. Li, F. Zhang, H. Wang, and J. Liu, "The characteristics and self-time-delay synchronization of two-time-delay complex Lorenz system,” Journal of the Franklin Institute, vol. 356, no. 1, pp. 334-350, 2019.

[5] F. Zhang, K. Sun, Y. Chen, H. Zhang, and C. Jiang, "Parameters identification and adaptive tracking control of uncertain complex-variable chaotic systems with complex parameters,” Nonlinear Dynamics, vol. 95, no. 4, pp. 3161-3176, 2019.

[6] F. Zhang, M. Li, S. Leng, and J. Liu, "Linear Correlation of Complex Vector Space and Its Application on Complex Parameter Identification,” Journal of Qilu University of Technology, vol. 33, no. 1, pp. 70-73, 2019.

[7] M. Mackey and L. Glass, "Oscillation and chaos in physiological control systems,” Science, vol. 197, no. 4300, pp. 287-289, 1977.

[8] Pyragas and K., "Transmission of Signals via Synchronization of Chaotic Time-Delay Systems,” International Journal of Bifurcation \& Chaos, vol. 8, no. 09, pp. -, 1998.

[9] J. Sun, "Global synchronization criteria with channel time-delay for chaotic time-delay system,” Chaos Solitons \& Fractals, vol. 21, no. 4, pp. 967-975, 2004.

[10] J. Tang, C. Zou, S. Wang, L. Zhao, and P. Liu, "Chaos synchronization of chen systems with time-varying delays," International Journal of Bifurcation \& Chaos, vol. 22, no. 08, p. 8, 2012.

[11] F. Zhang, S. Liu, and W. Yu, "Characteristics of complex Lorenz chaotic system with delay and its self-delay synchronization,” Acta Physica Polonica B, vol. 62, no. 22, pp. 220505-9, 2013.

[12] A. Wolf, J. B. Swift, H. L. Swinney, and J. A. Vastano, "Determining Lyapounov exponents from a time series," Physica D Nonlinear Phenomena, vol. 16, no. 3, pp. 285-317, 1985.

[13] J. K. Hale, Functional Differential Equations. 1971.

[14] N. Khrustova, G. Veser, A. Mikhailov, and R. Imbihl, "Delay-Induced Chaos in Catalytic Surface Reactions: NO Reduction on Pt(100)," Physical Review Letters, vol. 75, no. 19, pp. 3564-3567, 1995.

[15] B. P. Bezruchko, A. S. Karavaev, V. I. Ponomarenko, and M. D. Prokhorov, "Reconstruction of time-delay systems from chaotic time series,” Physical Review E Statistical Nonlinear \& Soft Matter Physics, vol. 64, no. 5 Pt 2, p. 056216, 2001.

[16] L. Chen, Y. Chai, and R. Wu, "Lag projective synchronization in fractional-order chaotic (hyperchaotic) systems,” Physics Letters A, vol. 375, no. 21, pp. 2099-2110, 2011.

[17] J. Min, W. Hong, and S. Bo, "Control and synchronization of fractional order delay complex Lorenz chaotic systems," Application Research of Computers, vol. 34, no. 3, pp. 730-733, 2017.

[18] A. C. Fowler, J. D. Gibbon, and M. J. McGuinness, “The complex Lorenz equations,” Physica D Nonlinear Phenomena, vol. 4, no. 2, pp. 139-163, 1982. 